\title{
Urban Tree Classification Using Discrete-Return LiDAR and an Object-level Local Binary Pattern Algorithm
}

\author{
Omer Saud Azeez ${ }^{1}$, Biswajeet Pradhan ${ }^{2 *}$, Ratiranjan Jena \\ ${ }^{1}$ Department of Civil Engineering, Faculty of Engineering, University Putra Malaysia, 43400, \\ UPM, Serdang, Malaysia \\ ${ }^{2}$ The Centre for Advanced Modelling and Geospatial Information Systems (CAMGIS), Faculty of \\ Engineering and Information Technology, University of Technology Sydney, 2007 NSW, \\ Australia
}

*Email. Biswajeet.Pradhan@uts.edu.au; biswajeet24@gmail.com 


\title{
Urban Tree Classification Using Discrete-Return LiDAR and an Object-level (Local Binary Pattern Algorithm)
}

\begin{abstract}
Urban trees have the potential to mitigate some of theharm brought about by rapid urbanization and population growth, as well as serious environmental degradation (e.g. soil erosion, carbon pollution and species extirpation), in cities. This paper presents a novel urban tree extraction modelling approach that uses discrete laser scanning point clouds and object-based textural analysis to (1) develop a model characterised by four sub-models, including (a) height-based split segmentation, (b) feature extraction, (c) texture analysis and (d) classification, and (2) apply this model to classify urban trees. The canopy height model is integrated with the objectlevel local binary pattern algorithm (LBP) to achieve high classification accuracy. The results of each sub-model reveal that the classification of urban trees based on the height at 47.14 (high) and $2.12 \mathrm{~m}$ (low), respectively, while based on crown widths were highest and lowest at 22.5 and $2.55 \mathrm{~m}$, respectively. Results also indicate that the proposed algorithm of urban tree modelling is effective for practical use.
\end{abstract}

Keywords. Urban tree classification; LBP; Object-based; Remote sensing; GIS; LiDAR

\section{Introduction}

Over $50 \%$ of the world's population lives in urban areas, and research indicates that this percentage will approach $75 \%$ by 2050 (Roberts, 2011). Land cover classification is non-specific and differs from vegetation classification. The classification of the tress in computer-based remote sensing studies is challenging due to factors such as density, shadow, size, and transition zones.

Urban trees supply a wide range of services for residents, such as carbon pollution mitigation and air quality improvement in urban environments (Janhäll, 2015), attenuation of storm-water flooding (Miller, 2015), energy conservation (Low et al., 2005), noise reduction (Dwyer et al., 1992) and habitat provision for urban wildlife (McPherson et al., 2011). An urban tree inventory is often needed for proper planning and management to maximise these benefits. Several methods, including per-pixel and object-based classification can be used to prepare an urban tree inventory (Tehrany et al., 2014; Jebur et al, 2014; Sibaruddin et al., 2018). Different datasets are used to 
extract urban data, such as high-resolution satellite images, aerial photos and light detection and ranging (LiDAR) point clouds and hyperspectral images, with different levels of precision.

While Landsat Thematic Mapper (LTM) and SPOT are considered high-resolution visible satellite imaging techniques, they have been proven to be inadequate for differentiating species-level trees in some classification studies (Kalliola and Syrjanen, 1991; Harvey and Hill, 2001). The accuracy of these techniques at the level of species-wise thematic information extraction has been reported to be $40 \%$ or less (Czaplewski and Patterson, 2003). A wide range of high-resolution remote sensing images, such as multispectral airborne and space-borne imagery with a spatial resolution of $1 \mathrm{~m}$ or more, have become readily available in recent years. The importance of high-spatial resolution imagery must be assessed to achieve automated vegetation classification (Ehlers et al., 2003). High-spatial resolution imagery was initially used for urban feature extraction (Jensen and Cowen, 1999; Benediktsson et al., 2003). High-resolution images are also used to detect spectral signature proximity and capture difficulties in feature texture during vegetation mapping (Carleer and Wolff, 2004).

LiDAR is a cutting-edge remote sensing technique that utilises laser pulses to accurately represent the earth's surface. LiDAR data are often used in elevation models due to their higher accuracy and faster data capturing process compared with traditional field survey techniques (Lefsky et al., 2002). LiDAR data can also be utilised for feature extraction. Many types of features, such as buildings, roads and trees, can be extracted through LiDAR data (Mahmoud et al., 2011; Tehrany et al., 2014; Jebur et al, 2014; Sibaruddin et al., 2018; Ghasemi et al., 2018). The most recent application of LiDAR is urban tree extraction. Urban trees have significant importance in urban planning due to their many applications, such as noise mitigation, traffic emission reduction, temperature mitigation in urban areas and optimisation of landslide conditioning factors. LiDARderived parameters have also been used in natural hazard and risk assessment (Jebur et al., 2014; Jebur et al., 2015; Abdulwahid et al., 2017; Liu et al., 2017; Azeez et al., 2018, 2019; Jena et al., 2018, 2019; Pradhan et al., 2018).

\section{Literature review}

Urban tree detection and classification methods based on traditional approaches, such as ground surveyes, are time-consuming, expensive and require considerable effort. Therefore, alternative methods, such as remote sensing, have gradually gained favour amongst researchers due to their lower cost compared with other approaches. Many studies based on remote sensing data have been conducted. Traditionally, Kettig and Landgrebe (1976) developed a spatial-spectral classifier 
known as the extraction and classification of homogeneous objects (ECHO) for object classification. Some researchers have adopted ECHO classification for land use or land cover classification with the knowledge of image interpretation and achieved significantly improved results (Gong and Howarth, 1990; Herold et al., 2003b). Kettig and Landgrebe (1976) stated that the logic of this ECHO method is that the size of a pixel is smaller compared with that of the objects of interest. However, this approach has not been extensively implemented for the classification of land cover; instead, TM and HVR are used as prevailing available data. An increasing number of studies have found that the object-based approach can handle high-resolution images. The LBP algorithm is applicable to land cover and urban tree classification due to its high classification accuracy (Yang et al., 1999). The Delaunay triangulation approach, which is characterised by image texture primitives, was used to classify treetops for Compact Airborne Spectrographic Imager NIR band with a resolution of $1.2 \mathrm{~m}$ (Bunting et al., 2006). However, they revealed that this technique outperforms conventional textures. Moreover, implementing this technique to broadleaf forests is unreasonable because identifying treetops through this technique is impossible. Some studies on Delaunay triangulation explain that the efficiency of LBP for highresolution remote sensing images is higher than that of conventional pixel-based approaches. Therefore, the LBP algorithm approach is highly suggested for urban tree classification.

Some of the most recent studies combining remote sensing data with different types of modelling approaches are described in this section. Schreyer et al. (2014) proposed a model by combining LiDAR data and high-resolution satellite images (QuickBird) with a spatial resolution of $60 \mathrm{~cm}$ to evaluate the carbon contents stored in trees using the canopy height model (CHM) in Berlin, Germany. These trees were then classified based on their carbon contents. The results were validated using field surveying, and an accuracy of $80.1 \%$ was obtained. This type of model represents a good tool for urban tree extraction, but limitations related to the spatial resolution of the QuickBird images exert a negative aspect on image classification. In a separate study, Zhang et al. (2015) presented a novel algorithm based on the integration of hyperspectral images and LiDAR data in order to automate the extraction of the urban trees. The developed model was implemented based on the relative height of the vegetation points and the digital terrain model used to extract the canopy height. Their results achieved a maximum accuracy of $84 \%$. The main drawback of this study, the Canopy Height Model CHM did not provide sufficiently good accuracy in urban tree extraction. 
Image classification based on advanced models, such as artificial intelligence algorithms, has recently been implemented. Liu et al. (2017) presented an approach based on the integration between fused LiDAR-hyperspectral images and the random forest model to evaluate urban trees in urban areas in British Columbia, Canada. Their results showed a significant improvement, i.e. an increase in accuracy from $70 \%$ to $91 \%$, in classification accuracy and trees extraction when the LiDAR data were combined with hyperspectral images. The main drawback of this approach, however, is the use of hyperspectral data, which requires high-quality computers and is timeconsuming for the classification of large areas.

Shojanoori et al. (2016) presented an approach based on the integration between pixel- and objectbased image classification and the maximum likelihood and support vector machine models to classify tree species. The classification results were compared with the results of OBIA, which adopts rule-based classification. Here, the OBIA based on a rule set showed a huge potential to extract tree species with high precision. However, this model lacks elevation data, which are useful in extracting tree canopies and tree classification based on height. Although many models can extract and classify trees species, statistical separability reduction occurs due to spectral variations among pixel-based traditional approaches. The salt-pepper effect is exhibited by the classification results, and reduced accuracy is obtained, which is classified differently from individual pixels from their surrounding features. To overcome the problem of H-resolution, some object- and pixelbased methods, including (a) low-pass filtering (image pre-processing) and texture analysis (Hill and Foody, 1994); (b) contextual classification (Gong and Howarth, 1992a); and (c) mode and morphological filtering, probabilistic relaxation and rule-based processing under post-processing (Gong and Howarth, 1992a; Shackelford and Davis, 2003), have been applied. Neighbourhood relationships have also been used to incorporate spatial information in these methods. In a separate study, Wen et Al. (2017) proposed a novel framework using a three-level (pixel-object-patch) approach to classify urban trees as several classes (i.e. park, roadside, and residential-institutional trees) in Wuhan and Shenzhen, China. Their results achieved an overall accuracy of $85 \%$, the user's and producer accuracy were reached higher than $80 \%$. The proposed framework allows users to map urban trees that can aid in supporting decision making related to urban ecosystems and policymakers. On the other hand, Huang et al. $(2018,2017)$ proposed a framework based on the integration of high-resolution imagery sources and three methods such as unsupervised convolutional neural network (UCNN), supervised latent Dirichlet allocation (sLDA) and the bagof-visual-words (BOVW) model in order to map tea gardens in different areas in China. The final results indicated that the UCNN outperformed the other methods. On the other hand, the results 
showed that the addition of textural features enhanced the precision of sLDA and BOVW, while they noticed that there is no effect on the UCNN.

Most of the previous studies are conducted based on traditional and machine learning algorithms. However, research on detailed vegetation or tree classification using high spatial resolution imagery or LiDAR data is scarce. Moreover, the accuracy obtained from previous studies is limited to $70 \%-80 \%$. Therefore, in the present study, an object-level local binary pattern (LBP) algorithm with a discrete return LiDAR is expected to improve the accuracy of urban tree classification using four sub-models. Firstly, the CHM model was developed by subtracting the DEM from DSM in the classification, leading to a successful urban tree classification. Secondly, the CHM image was filtered with a median filter; height-based split segmentation and spectral-difference segmentation must be applied before classification. Finally, the LBP algorithm is implemented to search for the optimal subset of features and the classification. Gradient-based features classification, such as the wavelet, HOG and COV, are less accurate than the LBP algorithm, which is sparse and has better computation ability.

The main contributions of this study include the integration of the CHM and object-level LBP algorithm to obtain high discriminative power, invariance to grey-scale changes, computational simplicity and excellent performance of urban tree classification. Thus, this study presents a novel approach based on the object-level LBP algorithm and LiDAR data to extract and classify urban trees.

\section{Study area and methodology}

\subsection{Overall methodology}

This section illustrates the overall methodology of the urban tree classification (Figure 1). An LBP algorithm is proposed to extract the texture information of a single urban tree. Firstly, the LiDAR point clouds were interpolated to construct a very high-resolution digital surface model (DSM) using the ArcGIS inverse distance weighting (IDW) interpolation method (Lu et al., 2008; Chen et al., 2012; Fanos et al., 2019). Then, the multiscale curvature algorithm was utilised to construct the digital elevation model (DEM) in the ArcGIS environment. Afterwards, the CHM was built using a simple subtraction process (i.e. DSM - DEM). A $3 \times 3$ median filter was applied to correct the CHM through ArcGIS filtering tools. Next, a height-based split segmentation was run to segment the CHM image in the Definiens eCognition environment. The segmentation results were

further refined by applying additional spectral difference segmentation and small object $(<130$ Pixels) removal within the constructed segments. 
Upon completion of segmentation, the texture pattern of each segment was generated using the LBP algorithm implemented in MATLAB R2016b. LiDAR returns extracted a uniform local binary pattern (LBP) from the image. We extracted LBP features from the images to encode their textural specifications. Then the process continues through gauge between the LBP features similarity by calculating the squared error in between them using MATLAB R2016b. Visualization of the squared error to compare the similar texture objects was obtained. Then we extracted the unnormalized LBP features, which can be used for custom normalization. The next step includes reshaping and normalising the LBP features. Additional spatial, spectral, textural, and structural features were extracted for each segment for classification and modelling using MATLAB R2016b. The relevant features were then selected by the support vector machine (SVM) feature selection method. Urban trees were classified using machine learning classification (MLC) methods and the selected relevant features. Finally, the classified segments were exported into GIS to calculate the tree parameters, including height, crown area, carbon stock and stem diameter in the ArcGIS environment. 


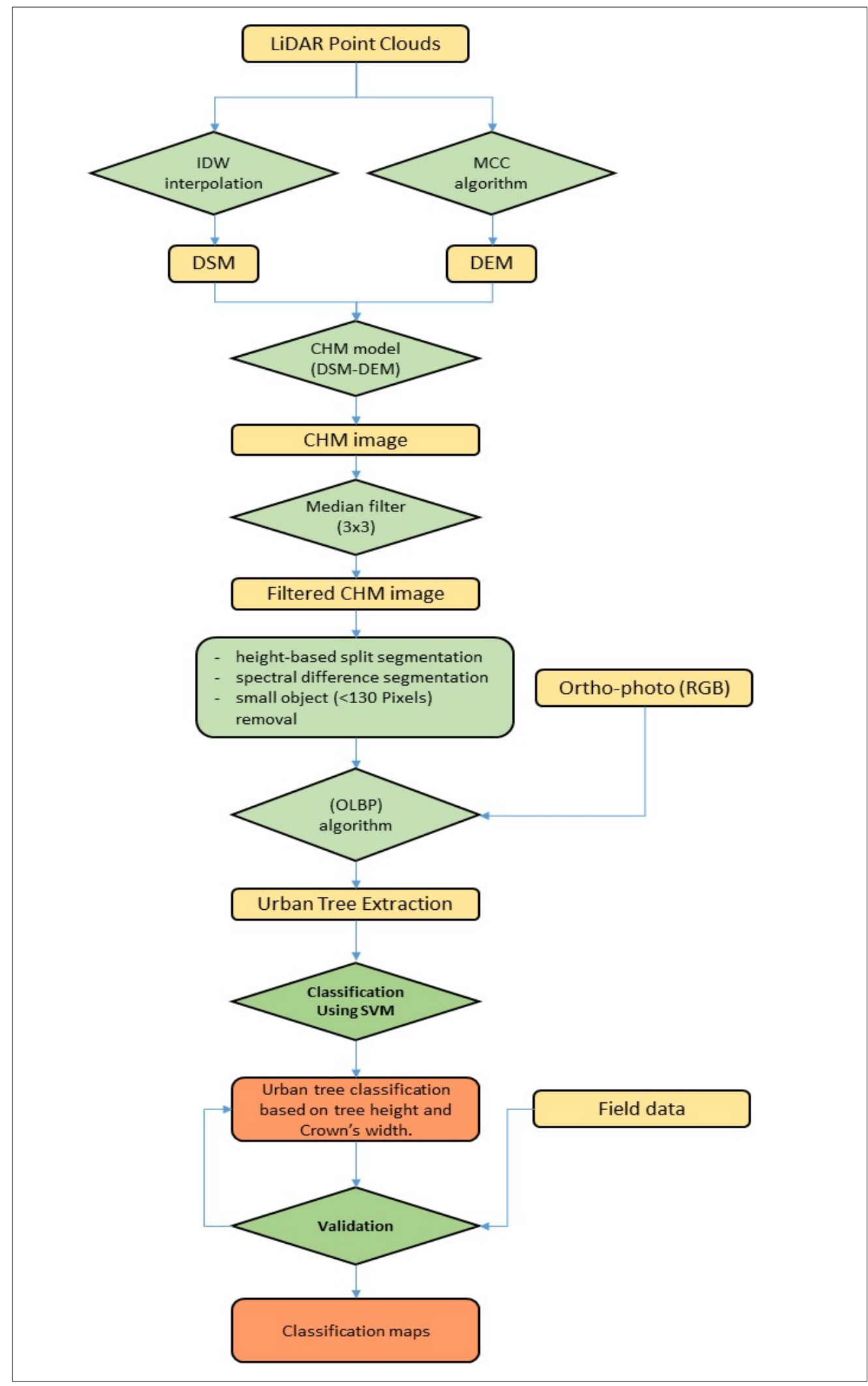

Figure 1. Overall methodology applied to this study. 


\subsection{Study area}

The current study was implemented in a complex urban area in the University Putra Malaysia within Serdang City in Selangor State, Malaysia. The study area is located between the longitude of $101^{\circ} 42^{\prime}-101^{\circ} 43^{\prime}$ and the latitude of $3^{\circ} 0^{\prime} 9^{\prime \prime}-3^{\circ} 0^{\prime} 27^{\prime \prime}$. The study area also contains many buildings and trees of various heights and types. The road network of the university is connected to the surrounding locations. The test sample surface area is approximately $1 \mathrm{~km}^{2}$ and illustrated in Figure 2. The dataset used in this study contains LiDAR point clouds and aerial photos. LiDAR data were collected using an airborne platform on March 8, 2015, with a scanning angle of $60^{\circ}$ and a camera angle of $\pm 30^{\circ}$. In addition, the LiDAR data density is 3-4 pts $/ \mathrm{m}^{2}$, and the highest and lowest elevations are 69 and $36 \mathrm{~m}$, respectively. Another dataset used in this study is aerial photos, which were collected by airborne platforms with a $10 \mathrm{~cm}$ spatial resolution and red/green/blue (RGB) bands.

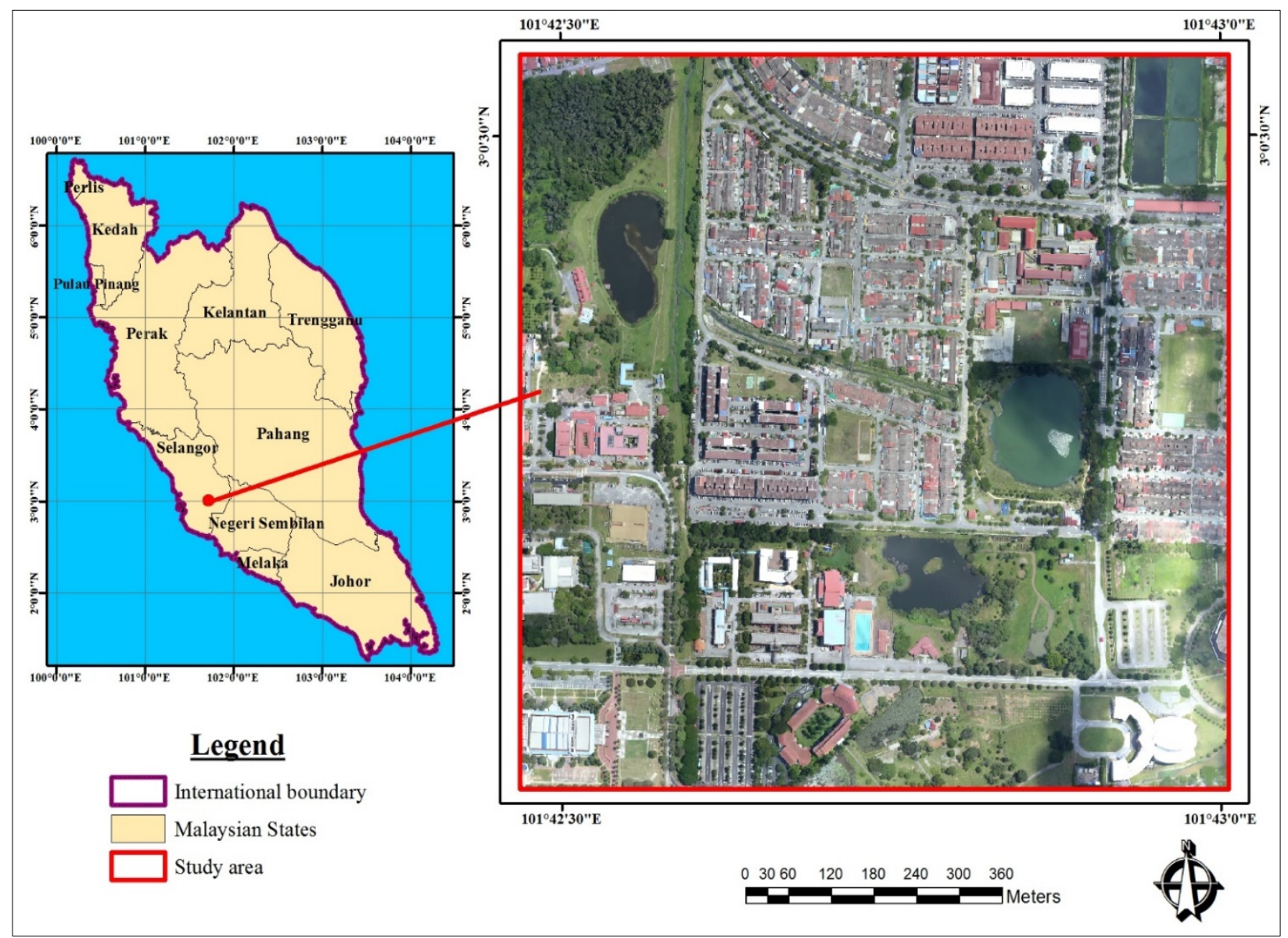

Figure 2. Study area map. 


\subsection{Canopy Height Model}

The CHM which is a representation of the tree canopy in raster form, was extracted from the airborne LiDAR data and used to detect all features on the ground in ArcGIS software. The model can be extracted based on the difference between DSM and DEM (Figure 3) and is defined as the shortest distance between the treetops and ground surface. Raw LIDAR data is a collection of mass points with XYZ coordinates. To generate DSM, the LiDAR data was filtered by selecting the first return and non-ground points. The point data must be interpolated into regular grid data. There are several surface interpolation methods, such as inverse distance weighted interpolation, kriging, polynomial regression, etc. Our purpose was to extracting buildings rather than constructing a smooth surface, therefore, an inverse distance weighted interpolation method was chosen because it will preserve the sharp difference between buildings and their surrounding ground. Firstly, in the LiDAR processing phase, the classified point clouds were converted to the ground and nonground points using the IDW and MCC algorithms, respectively (Pirotti, 2010). Successive gridding of the resulting DEM yields a $0.5 \mathrm{~m}$ resolution raster map. Some CHMs include the results of trees overlapping with buildings. Therefore, enhancement and filtering processes should be applied to the input data to obtain accurate results. Statistically, the CHM model can be represented as shown below:

$$
C H M=D S M-D E M
$$

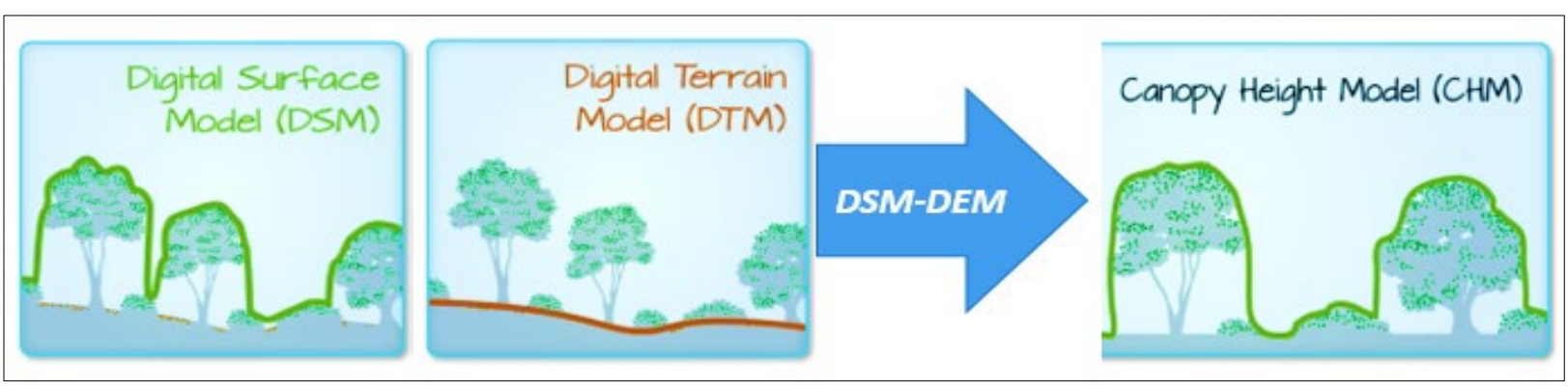

Figure 3. Schematic representation of the CHM. 


\subsection{Median Filter}

Median filtering is a nonlinear filtering approach frequently used to reduce and remove noise from images. Such a technique could be adopted as a standard pre-process to enhance later analyses, such as boundary detection in images. This approach has become common in digital image enhancement due to its ability to preserve features edges and remove noise under normal conditions. Similar to the mean filter, the median filter considers each pixel in the image and then decides, by examining it, whether its nearby neighbours are representative of its surroundings. The median value depends on the sorting of the surrounding pixel values into a numerical sequence and the change in pixel value with the middle pixel values. Figure 4 shows an example of pixel calculation in the median filter using ArcGIS environment.

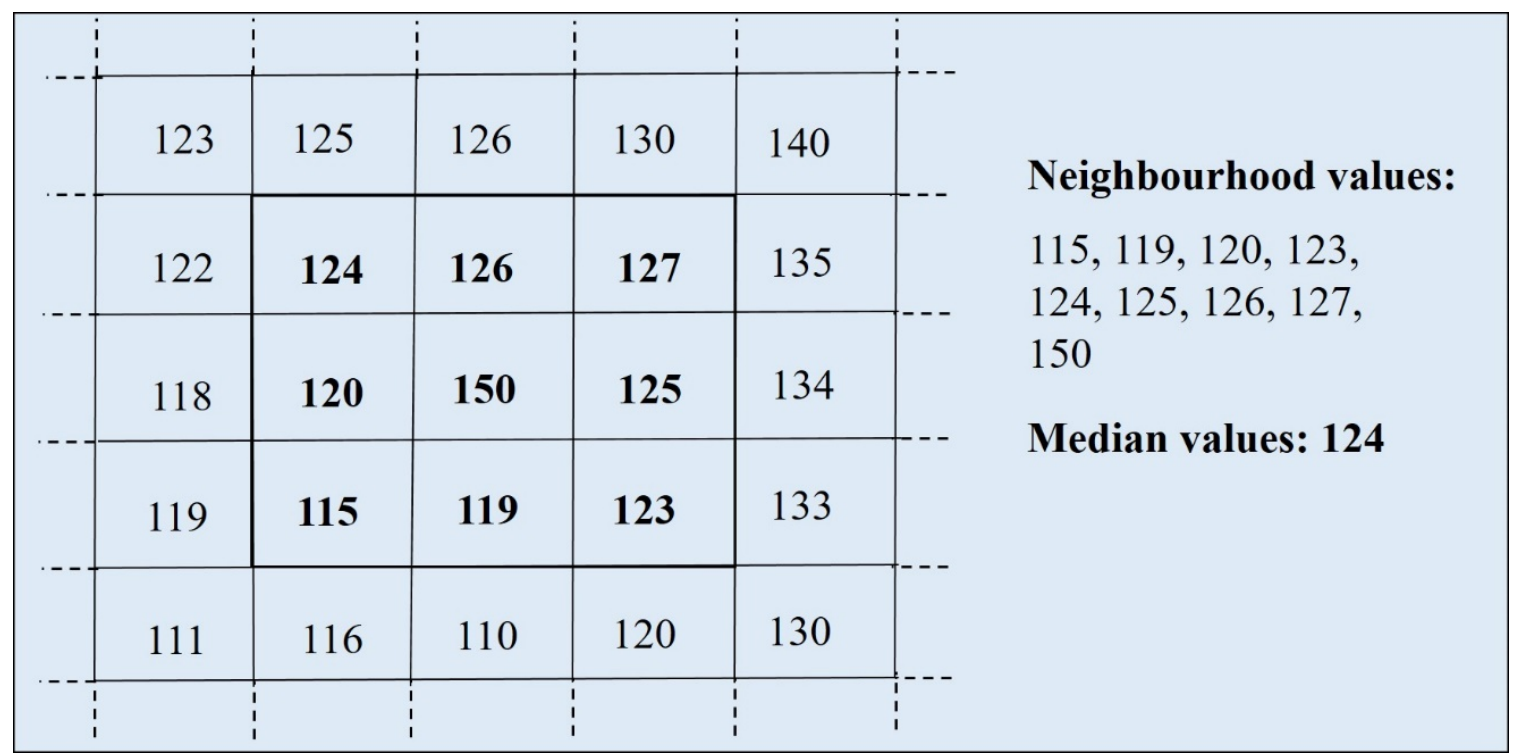

Figure 4. Median value calculation of a pixel neighbourhood.

\subsection{Image segmentation}

Spectral difference segmentation (SDS) is a merging algorithm in which adjacent objects with a spectral average lower than the given threshold, therefore the spectral differences will be merged to delineate final objects. This type of segmentation is used for further enhancement of the previous segmentation results. Height based split segmentation was performed, where some smooth crowns could be split effectively, and using the Full Lambda the Schedule algorithm could effectively merge the over-segmentation area, which guarantees the adequacy of the segmentation to reduce over-segmentation; The key point is that multiple segmentation steps are sometimes needed to produce objects most suitable to classification. 
Table 1 shows the feature specifications used in the segmentation, including the spatial characteristics extracted from the aerial photo (area, compactness, and roundness). The spectral specifications were also extracted from the aerial photo (RGB) bands using a Definiens eCognition environment. The height information was derived from the LiDAR data (first return elevation, last return elevation, intensity, CHM, number of points and maximum number of returns). In addition, novel information was extracted from the textural specifications containing grey-level cooccurrence matrices (GLCMs). Thus, the threshold achieved in the present study is the maximal spectral difference, which is 12 . Highly motivated by the study of Ostdijk et al. (2008), two segmentation types, namely, height-based split segmentation and spectral difference segmentation were adopted, and both approaches consistently helped obtain decent segmentation results across the datasets.

Table 1. Feature specifications

\begin{tabular}{|c|c|c|}
\hline No. & $\begin{array}{c}\text { Feature } \\
\text { Category }\end{array}$ & Feature \\
\hline 1 & \multirow{3}{*}{ Spatial } & Area \\
\hline 2 & & Compactness \\
\hline 3 & & Roundness \\
\hline 4 & \multirow{6}{*}{$\begin{array}{l}\text { LiDAR } \\
\text { Structure }\end{array}$} & First Return Elevation \\
\hline 5 & & Last Return Elevation \\
\hline 6 & & Intensity \\
\hline 7 & & $\mathrm{CHM}$ \\
\hline 8 & & Number of Points \\
\hline 9 & & Maximum Number of Returns \\
\hline 10 & \multirow{3}{*}{ Spectral } & Blue \\
\hline 11 & & Green \\
\hline 12 & & Red \\
\hline 13 & \multirow{8}{*}{ Textural } & GLCM Ang. $2^{\text {nd }}$ Moment (all dir.) \\
\hline 14 & & GLCM Contrast (all dir.) \\
\hline 15 & & GLCM Correlation (all dir.) \\
\hline 16 & & GLCM Dissimilarity (all dir.) \\
\hline 17 & & GLCM Entropy (all dir.) \\
\hline 18 & & GLCM Homogeneity (all dir.) \\
\hline 19 & & GLCM Mean (all dir.) \\
\hline 20 & & GLCM StdDev (all dir.) \\
\hline 21 & & Window size $(3 \times 3)$ \\
\hline
\end{tabular}

\subsection{LBP algorithm}

LBPs were introduced in 2002 by Ojala et al. (2002) as a texture descriptor. LBPs have been applied to many applications, including face recognition (Qi et al., 2015), facial expression (Chao et al., 2015) and wood recognition systems (Nasirzadeh et al., 2010). The main advantage of LBPs is that they are grey-scale and rotation invariant. The description of the LBP of a pixel is 
demonstrated by the threshold value of the $3 \times 3$ neighbourhood of the pixel against the central pixel, and the result is interpreted as a binary number. The binary code of the centre point was calculated using the following equation in MATLAB R2016b:

$$
\begin{gathered}
\operatorname{LBP}_{P, R\left(x_{c}, y_{c}\right)}=\sum_{i=0}^{P-1} 2^{i} \cdot S\left(g_{i}-g_{c}\right) \\
S\left(g_{i}-g_{c}\right)=\left\{\begin{array}{l}
1, g_{i}-g_{c} \geq 0 \\
0, g_{i}-g_{c}<0
\end{array}\right.
\end{gathered}
$$

where $\left(x_{c}, y_{c}\right)$ is the central pixel coordinates, $g_{c}$ is the central pixel grey value, $g_{i}$ is the value of its neighbours, $\mathrm{P}$ is the involved number of neighbours and $\mathrm{R}$ is the neighbourhood radius. Suppose the face image is of size $(M \times N)$. After identifying the LBP code of each pixel $\left(\mathrm{x}_{\mathrm{c}}, \mathrm{y}_{\mathrm{c}}\right)$, the structural and statistical approaches can be effectively combined by calculating the occurrence histogram.

$$
\begin{gathered}
H_{r}=\sum_{x_{c}=2}^{N-1} \sum_{y_{c}=2}^{M-1} f\left(\operatorname{LBP}_{P, R}\left(x_{c}, y_{c}\right), R\right) \\
f\left(L_{B} P_{P, R}\left(x_{c}, y_{c}\right), R\right)=\left\{\begin{array}{c}
1,\left(\operatorname{LBP}_{P, R}\left(x_{c}, y_{c}\right), R\right)=r \\
0, \text { otherwise }
\end{array}\right.
\end{gathered}
$$

Where the value of $r$ ranges from 0 to $2^{p}-1$.

\subsection{Support vector machine (SVM) classification}

Vapnik (1995) developed SVM, a non-linear classification model derived from machine learning techniques. SVM aims to determine an optimal separating hyperplane (maximizing the margin width) between two classes in a feature space. The training points near the hyperplane are called support vectors, and they are utilized for classification once the decision surface is obtained. The separating hyperplane is found as follows:

$$
y_{i}\left(w \times x_{i}+b\right) \geq 1-\xi_{i}
$$

where $\mathrm{w}$ is the coefficient vector that defines the hyper plane orientation in the feature space; $\mathrm{b}$ is the offset of the hyper plane from the origin; and $\xi_{\mathrm{i}}$ is the positive slack variables (Cortes and Vapnik, 1995). The optimal hyper plane is found by solving the following optimization problem (Jebur et al., 2014): 


$$
\begin{aligned}
& \text { Minimize } \sum_{i=1}^{n} \alpha_{i}-\frac{1}{2} \sum_{i=1}^{n} \sum_{j=1}^{n} \alpha_{i} \alpha_{j} y_{i} y_{j}\left(x_{i} x_{j}\right) \\
& \text { subject to } \sum_{i=1}^{n} \alpha_{i} y_{j}=0, \quad 0 \leq \alpha_{i} \leq C
\end{aligned}
$$

where $\alpha_{i}$ is the Lagrange multiplier and $\mathrm{C}$ is the penalty. For data classification, the following decision function is applied as follows:

$$
g(x)=\operatorname{sign}\left(\sum_{i=1}^{n} y_{i} \alpha_{i} x_{i}+b\right)
$$

In the case of non-linearly separating samples, the decision function (Equation 8) is rewritten as follows:

$$
g(x)=\operatorname{sign}\left(\sum_{i=1}^{n} y_{i} \alpha_{i} K\left(x_{i}, x_{j}\right)+b\right)
$$

In this process, the original data are transformed into a higher dimensional space using a nonlinear kernel function (K). The common K functions used with SVM in classification studies are linear, sigmoid, radial basis function, and polynomial (Bui et al., 2012). However, several studies have shown that radial basis function is more suitable for classification applications than other kernel functions (Pourghasemi et al., 2013; Xu et al., 2012). The SVM model was implemented based on supervised classification using MATLAB application.

\subsection{The Accuracy assessment}

Four quality measures, including root mean square (RMS) error, correctness, completeness, and overlay error, are used to assess the accuracy of the training and validation datasets. Correct extraction is represented by completeness, whilst RMS error is related to geometrical accuracy (Lee et al., 2003; Dehnavi et al., 2015; Mojaddadi et al., 2017; Naghibi et al., 2017). Area difference and overlay errors are used to determine the shape similarity between the trees (Azeez et al., 2018). We collected GTPs using a random method of data collection approach. The accuracy of the result was conducted by using the confusion matrix depending on Test samples 552 samples, 
a confusion matrix is a summary of prediction results on a classification problem. The number of correct and incorrect predictions are summarized with count values and broken down by each class. This is the key to the confusion matrix. The confusion matrix shows the ways in which your classification model is confused when it makes predictions (Table 2). It gives us insight not only into the errors being made by a classifier, but more importantly the types of errors that are being made. $a$ is the number of correct predictions that an instance is negative, $b$ is the number of incorrect predictions that an instance is positive, $\mathrm{c}$ is the number of incorrect predictions that an instance negative, and $\mathrm{d}$ is the number of correct predictions that an instance is positive.

Table 2. Confusion matrix for two classes.

\begin{tabular}{|c|c|l|c|}
\hline \multirow{2}{*}{} & \multicolumn{2}{|c|}{ Predicted } \\
\cline { 2 - 3 } \multicolumn{2}{|c|}{} & Negative & Positive \\
\hline \multirow{2}{*}{ Actual } & Negative & A & B \\
\cline { 2 - 4 } & Positive & C & D \\
\hline
\end{tabular}

Several standard terms have been defined for the 2 class matrix:

- The accuracy $(A C)$ is the proportion of the total number of predictions that were correct. It is determined using the equation: $\quad A C=\frac{a+d}{a+b+c+d}$

The recall or true positive rate $(T P)$ is the proportion of positive cases that were correctly identified, as

$$
\text { calculated using the equation: } T P=\frac{d}{c+d}
$$

- The false positive rate $(F P)$ is the proportion of negative cases that were incorrectly classified as positive, as calculated using the equation: $F P=\frac{b}{a+b} \quad[3]$

The true negative rate $(T N)$ is defined as the proportion of negative cases that were classified correctly, as calculated using the equation: $T N=\frac{a}{a+b}$ 
- The false negative rate $(F N)$ is the proportion of positive cases that were incorrectly classified as negative, as calculated using the equation: $F N=\frac{c}{c+d}$

- Finally, precision $(P)$ is the proportion of the predicted positive cases that were correct, as calculated using the equation: $\quad P=\frac{d}{b+d}$

The accuracy determined using equation 1 may not be an adequate performance measure when the number of negative cases is much greater than the number of positive cases (Kubat et al., 1998). Suppose there are 1000 cases, 995 of which are negative cases and 5 of which are positive cases. If the system classifies them all as negative, the accuracy would be $99.5 \%$, even though the classifier missed all positive cases. Other performance measures account for this by including $T P$ in a product: for example, geometric mean (g-mean) (Kubat et al., 1998), as defined in equations 7 and 8, and F-Measure (Lewis and Gale, 1994), as defined in equation 9 . In equation $9, \mathrm{~F}$ has a value from 0 to infinity and is used to control the weight assigned to $T P$ and $P$. Any classifier evaluated using equations 7, 8, or 9 will have a measured value of 0 if all positive cases are classified incorrectly.

$$
\begin{aligned}
& g-\text { mean }_{1}=\sqrt{T P * P} \\
& g-\text { mean }_{2}=\sqrt{T P * T N} \\
& F=\frac{\left(\beta^{2}+1\right) * P * T P}{\beta^{2} * P+T P}
\end{aligned}
$$




\section{Results and discussion}

\subsection{Classification of urban tree}

\subsubsection{Results of CHM and segmentation}

The DEM map presents the highest and lowest elevations of 47.70 and $36.42 \mathrm{~m}$, respectively. The DSM provides the same resolution due to gridding by combining the triangulated surfaces of the first and unique return points. The highest and lowest heights are respectively indicated as 89.26 and $36 \mathrm{~m}$ in the DSM map. Further processing prior to the production of the CHM model is no longer necessary due to the good result obtained. The difference between the two models provides the CHM. The result of the CHM indicates a maximum height of $44.55 \mathrm{~m}$ (Figure 5).
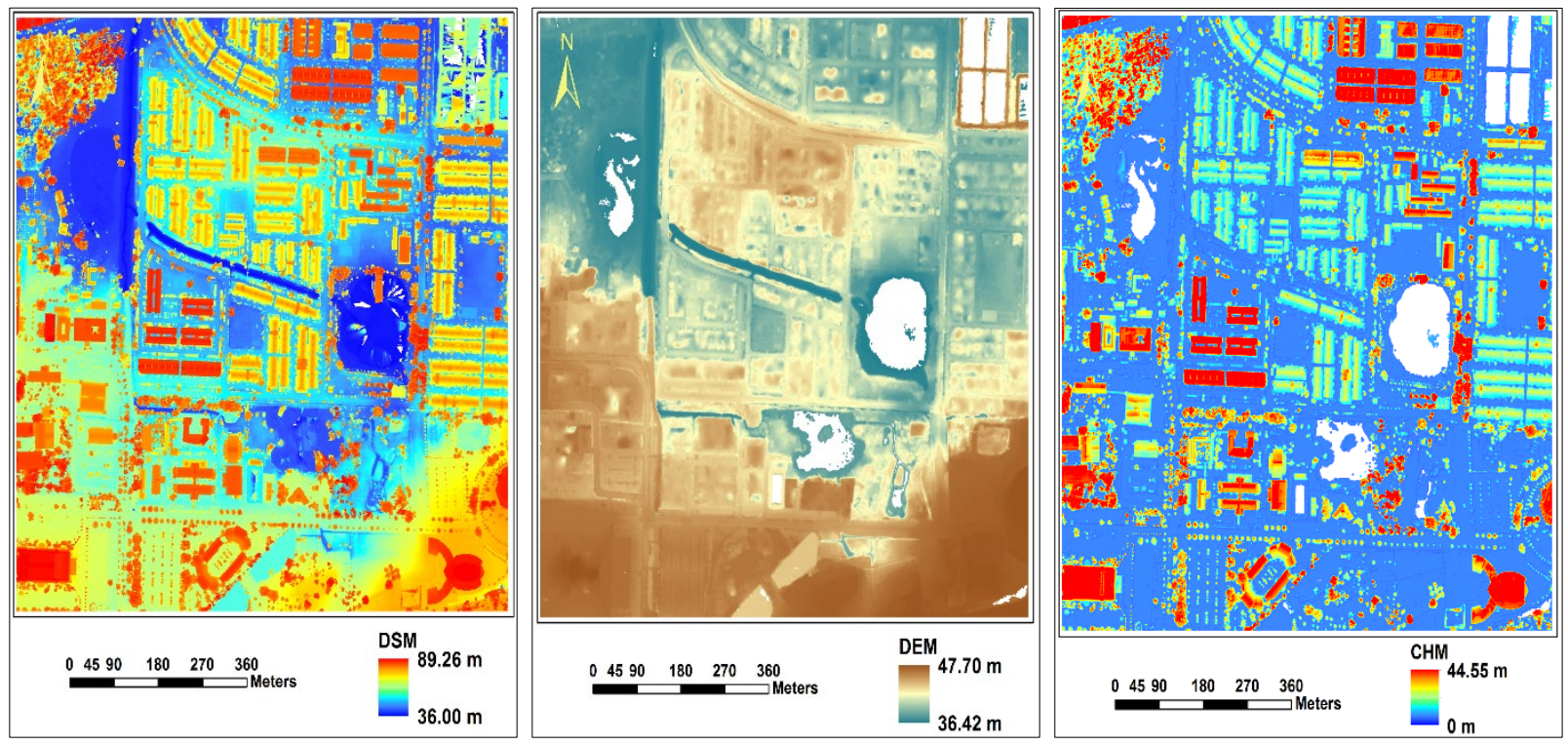

Figure 5. Results of CHM extraction.

In this study, the SDS algorithm merges the objects in the surrounding pixels with mean values lower than that of a specified threshold. Figure 6 illustrates the segmentation sample results using the multi-resolution and spectral difference segmentation algorithms. 


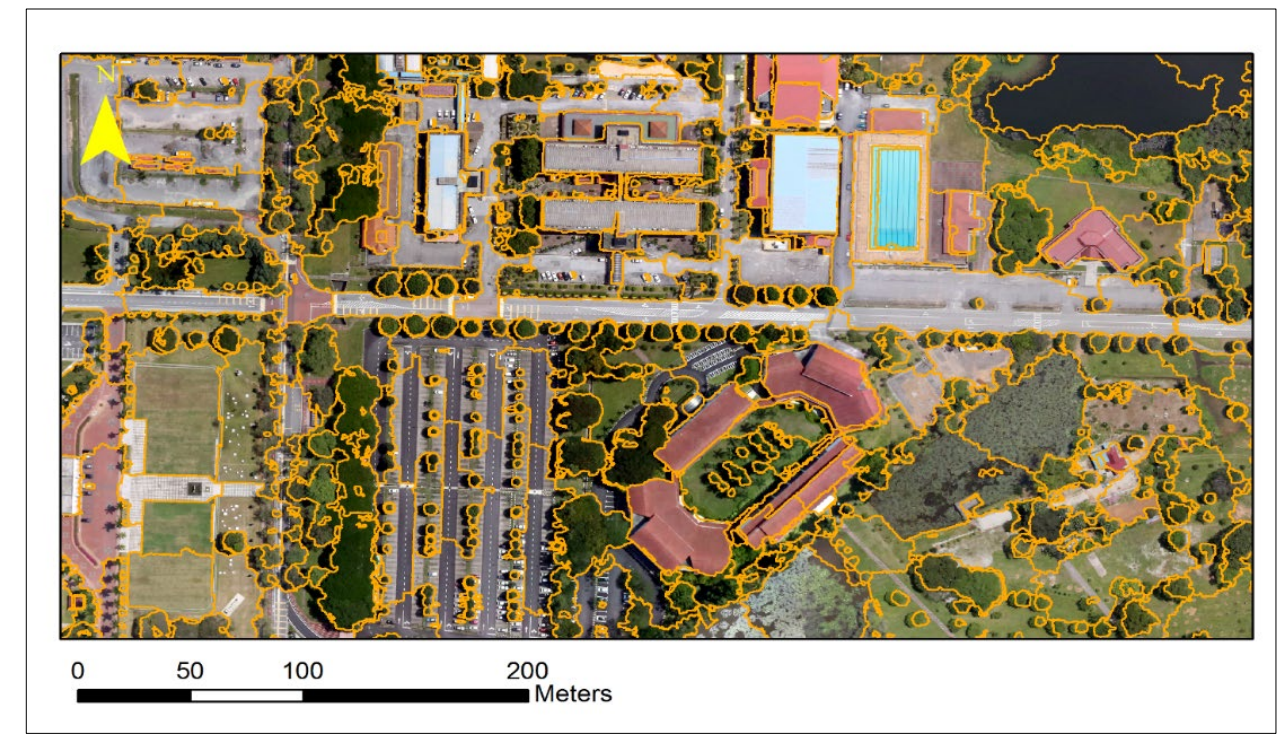

Figure 6. Results of spectral difference segmentation.

\subsubsection{Extraction of the LBPs of urban trees}

The ability of the LBP algorithm and LiDAR data to distinguish urban trees from buildings and other features by using various parameters, such as elevation, spectral and textural specifications, was examined in this study. The urban trees were extracted and classified using the proposed LBP model. Principally, the LBP algorithm was chosen to accord to the following criteria: 1) state-ofthe-art algorithm with significant accuracy, 2) appropriate for diversification of feature type classification and 3) preference to a few parameters to be used for classification. The proposed model showed improved segmentation performance compared with other models due to its application of the LBP of an urban tree. A new level of segmentation cannot be simply created by applying the spectral difference segmentation algorithm (Ryherd and Woodcock, 1996). Thus, SDS algorithm was applied to modify the segmentation over the previous segmentation, i.e. heightbased split segmentation. Using the previously generated segmentation, the new process tool was appended, and the spectral difference segmentation was processed. Segmentation adjustment of the scale parameters is necessary to best indicate the exact position of small homogenous urban tree patches, which are roughly in size. The criteria of segmentation comprise geometric indices and spectral homogeneity. The adopted spectral-based segmentation reduces the amount of data required for advanced processing and classification whilst preserving the information of spectral variation. Figure 7 illustrates the refinement of tree boundary extraction after implementation of the LBP model. 


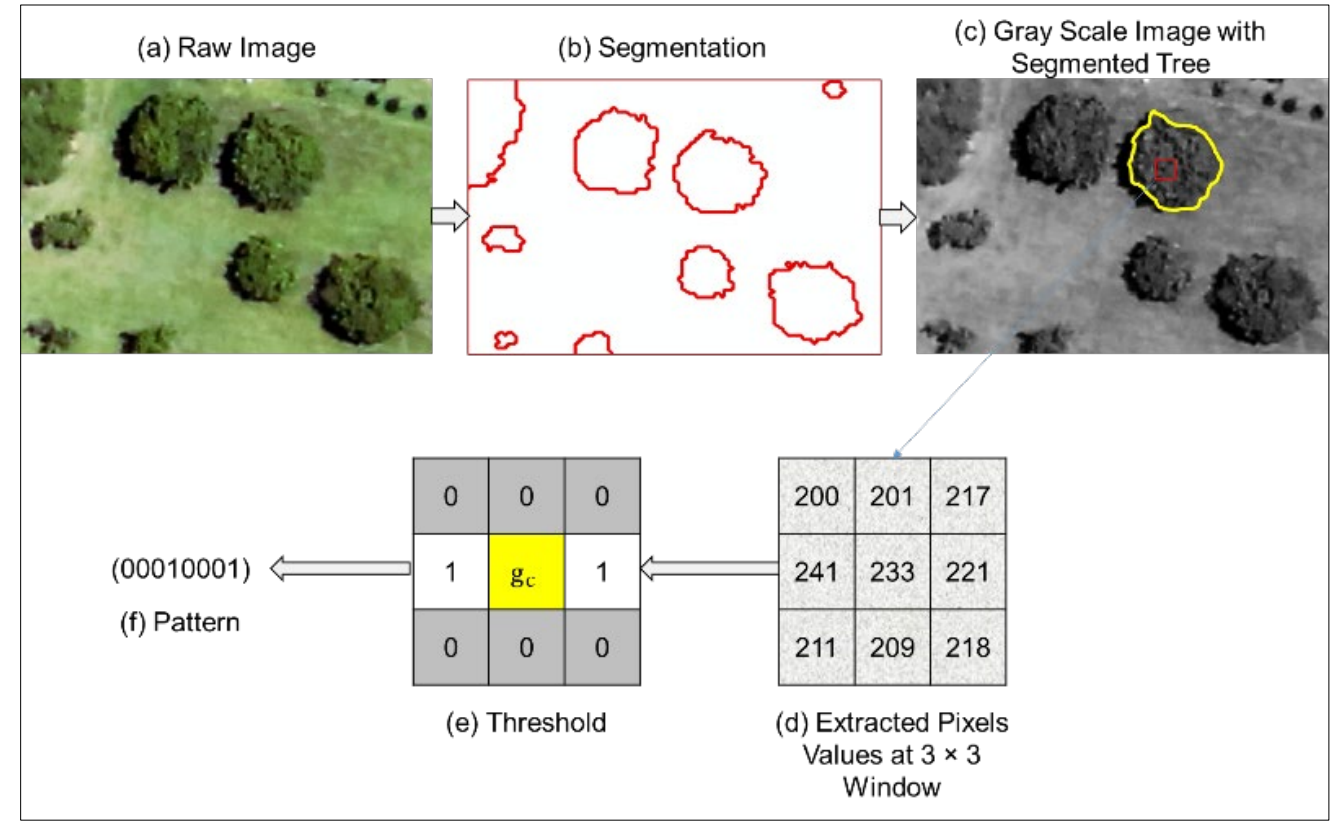

Figure 7. Extraction of the local binary pattern of an urban tree at the object level after segmentation.

\subsubsection{Classification of urban trees based on height and crown width}

Figure 8 shows the classification of urban trees based on height; the highest and lowest heights are 47.14 and $2.12 \mathrm{~m}$, respectively. The crown width is measured based on the OBLP algorithm, and the highest and lowest crown widths are found to be 22.5 and $2.55 \mathrm{~m}$, respectively.

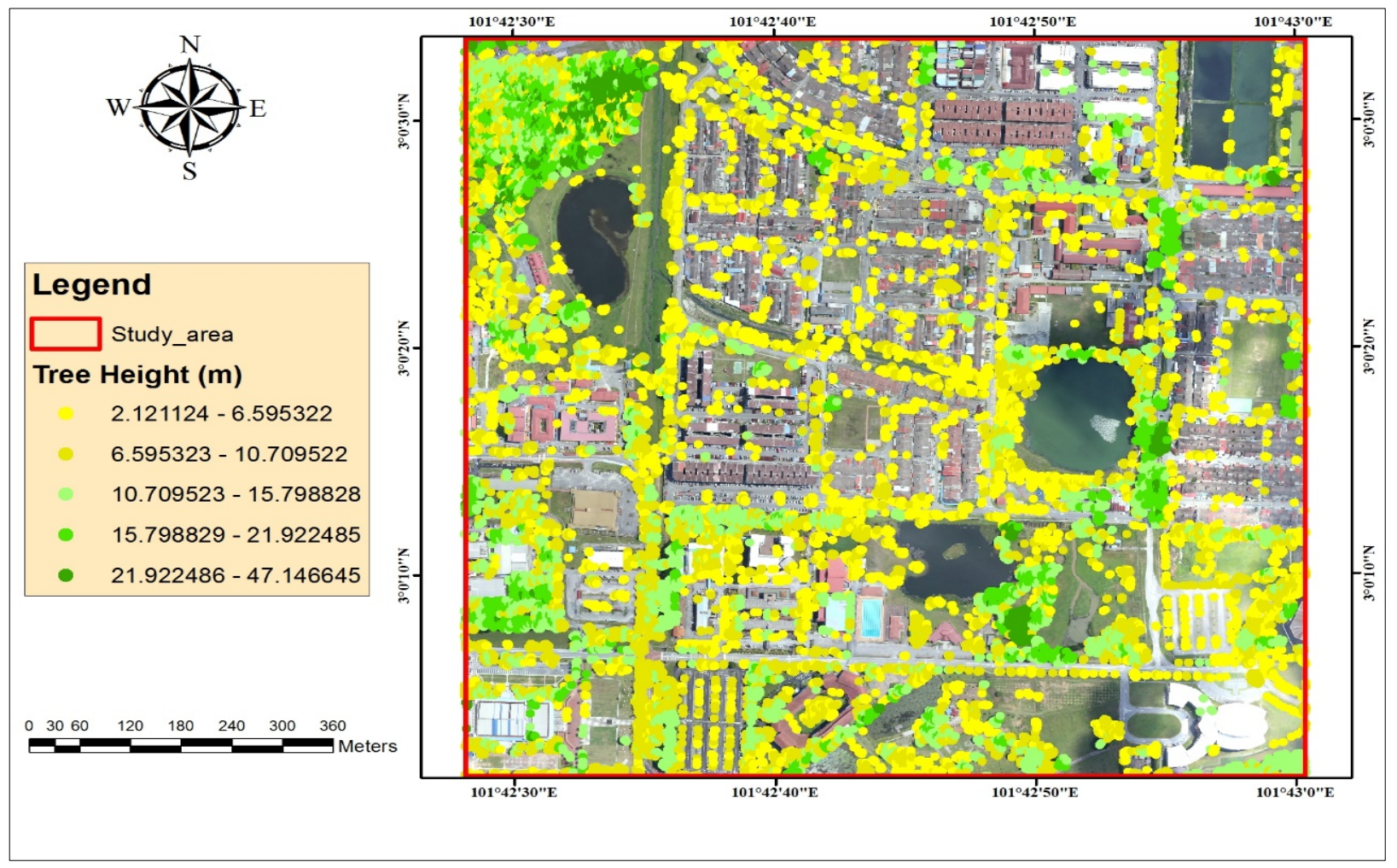


Figure 8. Urban tree classification based on tree height.

In this study, the objects based on LBP classification, which helps overcome salt-and-pepper effects arising from traditional pixel-based classification methods and is highly convenient. Amongst the topographic, geometric, spectral and textural features of an object, topographic information provides primary support, which is the most important feature of urban tree canopy or vegetation classification at this scale of spatial study. Therefore, in the study area predominantly for environment-dependent alliances, the urban trees were classified based on height. No significant contribution to urban tree classification of new geometric features was found. Researchers have used the hierarchical classification scheme to improve accuracy predominantly because optimal features are selected in a wide range of categories during classification. In some studies, pixel-based methods achieved an accuracy of 15\%-40\%. Pixel-based MLC, in particular, achieves significantly high accuracy due to its satisfactory classification performance with smallsized samples. According to our results, the LBP approach is more robust than the pixel-based MLC technique because of the specific characteristics of urban tree classification in the study location.

Segmentation was performed in MATLAB because of its coding advantage, and the relevant features were selected using the SVM and classified using the MLC algorithm to achieve high accuracy. LBP classification was conducted with all the selected attribute features. From all features, 20 features with four categories were selected for classification, and numerous overlaps were found. Table 3 shows the confusion matrix and the classification accuracy for each class. The urban tree accuracies varied considerably. In addition to the objective similarity of spectral characteristics, low accuracy may result from the following: (a) small sample size, such as those in Gorse and Cordgrass; (b) understory vegetation, such as mixed-vegetation types; and (c) species alliance composed of a dominant and minor species.

The results of the LBP classification approach are presented as raster maps in Figures 8 and 9 . The calculated accuracy was based on the correctly classified number of pixels for each class. The overall and average accuracies of LBP classification are described in the accuracy assessment and may be low if the pixel-based MLC or any traditional methods are applied. The accuracy assessment results can then be compared and analysed (Figures 8 and 9). Our findings illustrate that the LBP algorithm is significantly good in classifying urban trees with small sample sizes. 


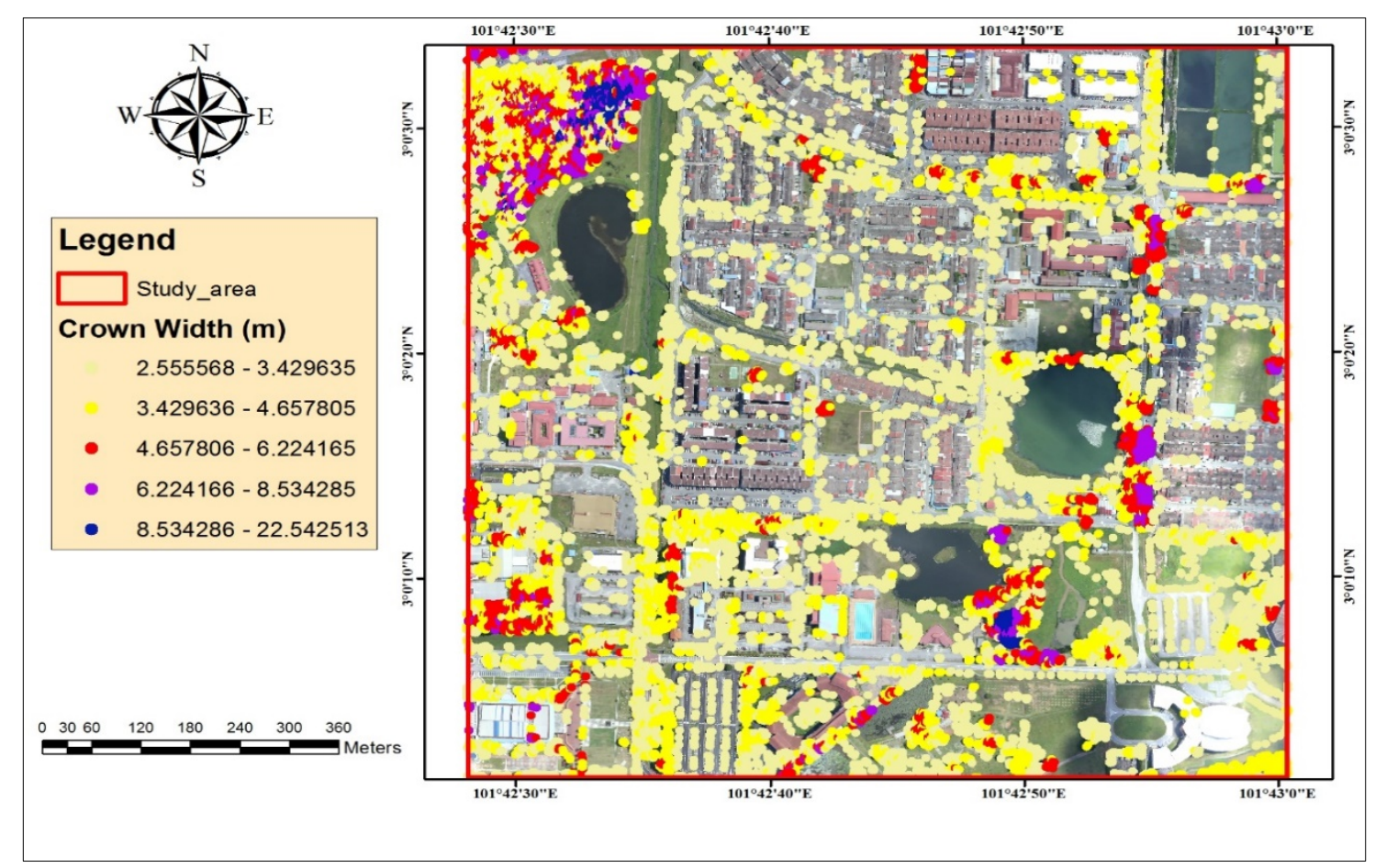

Figure 9. Urban tree classification based on crown width.

Finally, OBLP classification may be an excellent alternative to traditional pixel-based classification methods. Contiguous pixel groups should be analysed as objects as an alternative to using the classification unit of the conventional pixel-based approach to solving H-resolution issues and the salt-and-pepper effect (Yu et al., 2006). Reduction of local spectral variation can generally be conducted through groups of contiguous pixels caused by gaps, crown textures, and shadows (Yu et al., 2006). For further classification, spatial properties, such as shape and size roundness, along with spectral properties, can be employed as features for spectrally homogeneous segments of study location images. Spatially adjacent pixels are initially categorised into spectrally homogenous objects, and then the classification of objects is performed, which is the basic idea of object-based classification. This method may be perfect for the preferred characteristic of urban trees with few or no dominant trees. In this study, canopy shape does not create difficulty, therefore providing good results than that of other methods.

\subsection{Accuracy Assessment}

Validation of the data was conducted based on comparisons between the classified images and the ground truth data collected in the field. According to the confusion matrix, the overall accuracy of the proposed method is $92 \%$, and the Kappa index is 0.9012 . Table 3 shows the accuracy assessment results. 
Table 3. Confusion matrix and accuracy values of classification

\begin{tabular}{|c|c|c|c|c|c|c|c|}
\hline \multicolumn{4}{|c|}{ Ground Truth (Pixels) } & \multicolumn{4}{c|}{ Ground Truth (Percent) } \\
\hline Class & Buildings & Trees & Total & Class & Buildings & Trees & Total \\
\hline Unclassified & 289 & 36 & 325 & Unclassified & 3.846 & 0.13 & 23.56 \\
\hline Buildings & 7152 & 0 & 7152 & Buildings & 96.1 & 0 & 41.93 \\
\hline Trees & 4 & 5515 & 5519 & Trees & 0.054 & 99.87 & 36.07 \\
\hline Total & 7445 & 5551 & 12996 & Total & 100 & 100 & 100 \\
\hline
\end{tabular}

The total number of extracted trees is 1729 , and the original number of trees is 1839 . In addition, the completeness is $94 \%$; the RMSE is $0.2 \mathrm{~m}$ and the overlay error is $12 \%$.

\subsection{Comparison with traditional methods}

We compared the obtained results with other methods such as Neural Network (NN) model and Maximum Likelihood model, and we achieved 90.5 and 89.7 for NN and Maximum Likelihood, respectively. On the other hand, Kappa coefficients were recorded 0.88 and 0.85 . Therefore, the SVM classification was achieved the highest accuracy among these models. Then we generated a thematic map for the study area based on the final accuracy assessment. Figure 10 shows the accuracy comparison between the three models (neural network, SVM and maximum likelihood models).

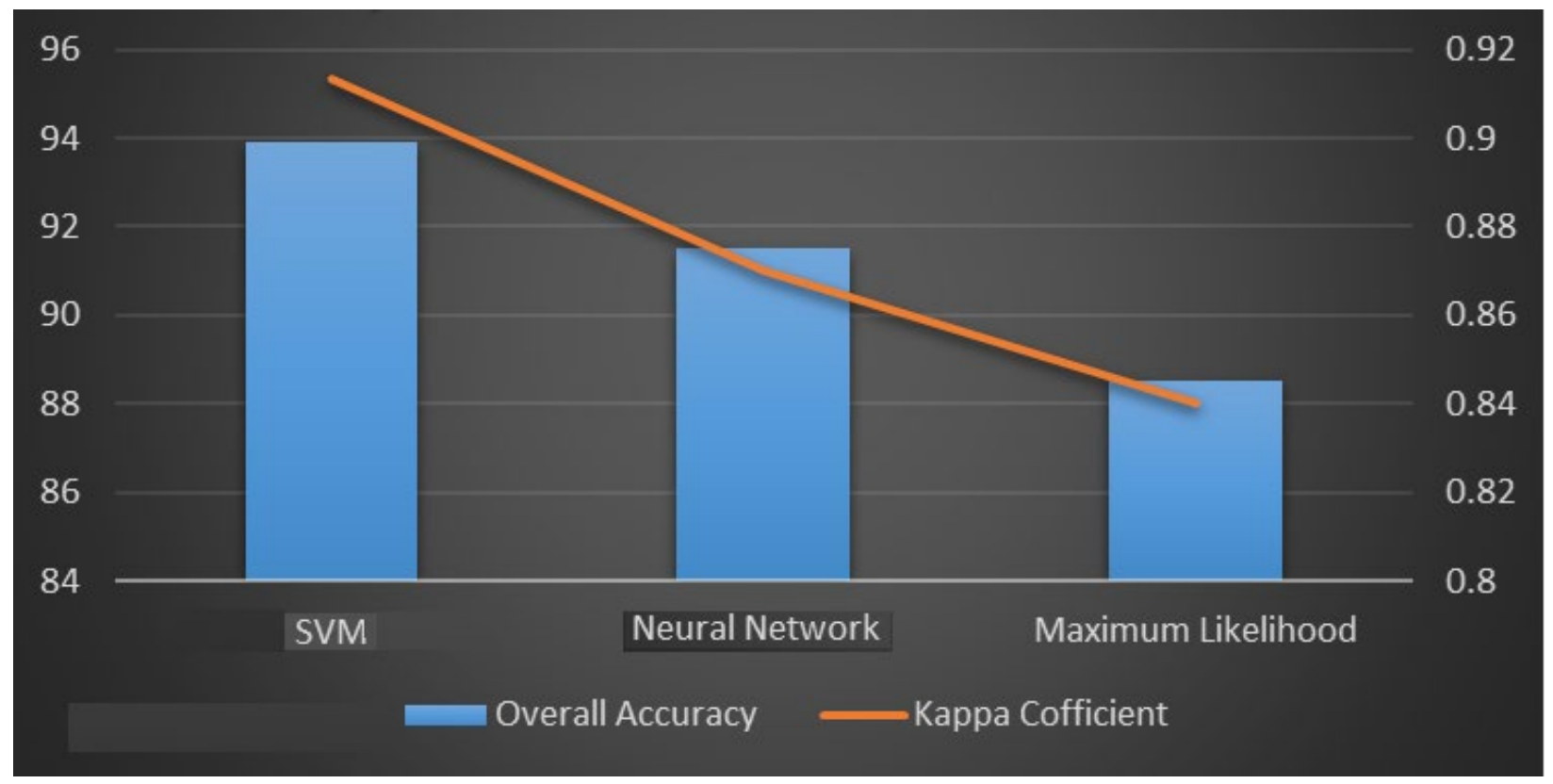

Figure 10. Comparison with traditional models 


\section{Conclusion}

The main objective of this study is to develop a novel modelling approach to classify urban trees and test the ability of the proposed LBP algorithm at the object level. The LBP algorithm was utilised in this study and applied after the segmentation process. The results illustrate that urban trees are characterised by deformations in their shapes. These deformations were refined by using the LBP algorithm, which is employed to improve the segmented objects based on different parameters, such as textural information and canopy height.

Different modelling approaches were used in this study for the exact analysis of positioning and extraction of tree and height measurement. Urban forest environments are generally heterogeneous in size and shape. This method is not expected to be accurate in all contexts, but an alternative to traditional methods that may work better in different situations is provided. The ideal context of this model is significant, and its application is not necessarily regularly spaced trees. The model can be applied to dominant layers and the canopy of ellipsoid-shaped trees. Embedded canopies within a clustered spatial distribution can be detected using the developed approach. The important parameter is the kernel and canopy size. While a large window filter provides low correlations that can handle multiple canopies, small windows produce noise effects and overestimate the tree position. Therefore, the filter should be chosen based on the expected output.

The presented dataset notably contains duplications between buildings and urban trees. However, the proposed model still manages to present refined boundaries of urban trees. In future work, the model will be extended to extract 3D tree models based on terrestrial LIDAR data, and parameters with complex shapes will be extracted and refined. Future investigations will also be performed to analyse the effect of the LiDAR point cloud and corresponding CHM resolution on the performance of the filter. Whether high point density and CHM resolutions could improve the results must also be investigated. The ability of the LBP algorithm to obtain expected results using a semi-automatic process is proven. In addition, some ground-truth surveys are necessary and could be applied to control the obtained results, conducting an economical edge. On the other hand, the urban trees or building deformations in high-resolution images can be reduced by using multiview images as presented by (Huang, 2018 and Huang, 2017).

Acknowledgements: The authors acknowledge and appreciate the provision of airborne laser scanning data (LiDAR), satellite images and logistic support by the PLUS Berhad. In addition, the second author, Biswajeet Pradhan, gratefully acknowledges the financial support from the UPMPLUS industry project grant. This research is supported by the Centre for Advanced Modelling 
and Geospatial Information Systems CAMGIS) in the University of Technology Sydney (UTS) under grant number 321740.323930, Grant 321740.2232335, Grant 321740.2232357, Grant 321740.2232424, and Grant 321740.2232452.

\section{References}

Abdulwahid, W.M., \& Pradhan, B. (2017). Landslide vulnerability and risk assessment for multihazard scenarios using airborne laser scanning data (LiDAR). Landslides, 14(3),n10571076.

Azeez, O. S., Kalantar, B., Al-Najjar, H. A. H., Halin, A. A., Ueda, N., \& Mansor, S. (2018). Object boundaries regularization using the dynamic polyline compression algorithm. International Archives of the Photogrammetry, Remote Sensing \& Spatial Information Sciences, 42(4).

Azeez, O.S., Pradhan, B., Shafri, H.Z., Shukla, N., Lee, C.W., \& Rizeei, H.M. (2019). Modeling of CO Emissions from Traffic Vehicles Using Artificial Neural Networks. Applied Sciences, 9(2), 313.

Azeez, O., Pradhan, B., \& Shafri, H. (2018). Vehicular CO Emission Prediction Using Support Vector Regression Model and GIS. Sustainability, 10(10), 3434.Benediktsson, J.A., Pesaresi, M., \& Amason, K. (2003). Classification and feature extraction for remote sensing images from urban areas based on morphological transformations. IEEE Transactions on Geoscience and Remote Sensing, 41(9), 1940-1949.

Bunting, P., and Lucas, R. (2006). The delineation of tree crowns in Australian mixed species forests using hyperspectral Compact Airborne Spectrographic Imager (CASI) data. Remote Sensing of Environment, 101(2), 230-248.

Carleer, A., \& Wolff, E. (2004). Exploitation of very high-resolution satellite data for tree species identification. Photogrammetric Engineering \& Remote Sensing, 70(1), 135-140.

Chao, W. L., Ding, J. J., \& Liu, J. Z. (2015). Facial expression recognition based on improved local binary pattern and class-regularized locality preserving projection. Signal Processing, $117,1-10$.

Chen, F.W. and Liu, C.W., (2012). Estimation of the spatial rainfall distribution using inverse distance weighting (IDW) in the middle of Taiwan. Paddy and Water Environment, 10(3), 209-222. 
Czaplewski, R.L., \& Patterson, P.L. (2003). Classification accuracy for stratification with remotely sensed data. Forest Science, 49(3), 402-408.

Dehnavi, A., Aghdam, I.N., Pradhan, B., \& Varzandeh, M.H.M. (2015). A new hybrid model using step-wise weight assessment ratio analysis (SWARA) technique and adaptive neuro-fuzzy inference system (ANFIS) for regional landslide hazard assessment in Iran. Catena, 135, 2015, 122-148. https://doi.org/10.1016/j.catena.2015.07.020

Dwyer, J. F., McPherson, E. G., Schroeder, H. W., \& Rowntree, R. A. (1992). Assessing the benefits and costs of the urban forest. Journal of Arboriculture, 18, 227-227.

Ehlers, M., Gähler, M., \& Janowsky, R. (2003). Automated analysis of ultra high resolution remote sensing data for biotope type mapping: new possibilities and challenges. ISPRS Journal of Photogrammetry and Remote Sensing, 57(5-6), 315-326.

Fanos, A.M., \& Pradhan, B. (2019). A spatial ensemble model for rockfall source identification from high resolution LiDAR data and GIS. IEEE Access. 7, 74570 - 74585. Doi: 10.1109/ACCESS.2019.2919977

Ghasemi, K., Pradhan, B., \& Jena, R. (2018). Spatial Identification of Key Alteration Minerals Using ASTER and Landsat 8 Data in a Heavily Vegetated Tropical Area. Journal of the Indian Society of Remote Sensing, 46(7), 1061-1073.

Gong, P., \& Howarth, P. (1990). The use of structural information for improving land-cover classification accuracies at the rural-urban fringe. Photogrammetric engineering and remote sensing, 56(1), 67-73.

Gong, P., \& Howarth, P.J. (1992). Land-use classification of SPOT HRV data using a coverfrequency method. International Journal of Remote Sensing, 13(8), 1459-1471.

Harvey, K.R., \& Hill, G.J.E. (2001). Vegetation mapping of a tropical freshwater swamp in the Northern Territory, Australia: a comparison of aerial photography, Landsat TM and SPOT satellite imagery. International journal of remote sensing, 22(15), 2911-2925.

Herold, M., Liu, X., \& Clarke, K.C. (2003). Spatial metrics and image texture for mapping urban land use. Photogrammetric Engineering \& Remote Sensing, 69(9), 991-1001.

Hill, R.A., \& Foody, G.M. (1994). Separability of tropical rain-forest types in the TambopataCandamo Reserved Zone, Peru. International Journal of Remote Sensing, 15(13), 26872693.

Huang, X., Zhu, Z., Li, Y., Wu, B., \& Yang, M. (2018). Tea garden detection from high-resolution imagery using a scene-based framework. Photogrammetric Engineering \& Remote Sensing, 84(11), 723-731. 
Huang, X., Chen, H., \& Gong, J. (2018). Angular difference feature extraction for urban scene classification using ZY-3 multi-angle high-resolution satellite imagery. ISPRS Journal of Photogrammetry and Remote Sensing, 135, 127-141.

Huang, X., Wen, D., Li, J., \& Qin, R. (2017). Multi-level monitoring of subtle urban changes for the megacities of China using high-resolution multi-view satellite imagery. Remote Sensing of Environment, 196, 56-75.

Janhäll, S. (2015). Review on urban vegetation and particle air pollution-Deposition and dispersion. Atmospheric Environment, 105, 130-137.

Jebur, M.N., Mohd Shafri, H.Z., Pradhan, B., \& Tehrany, M.S. (2014). Per-pixel and objectoriented classification methods for mapping urban land cover extraction using SPOT 5 imagery. Geocarto International, 29(7), 792-806.

Jebur, M.N., Pradhan, B. \& Tehrany, M.S. (2014). Optimization of landslide conditioning factors using very high-resolution airborne laser scanning (LiDAR) data at catchment scale. Remote Sensing of Environment, 152, 150-165.

Jebur, M.N., Pradhan, B. and Tehrany, M.S. (2015). Manifestation of LiDAR-derived parameters in the spatial prediction of landslides using novel ensemble evidential belief functions and support vector machine models in GIS. IEEE Journal of Selected Topics in Applied Earth Observations and Remote Sensing, 8(2), 674-690.

Jena, R., \& Pradhan, B. (2018), October. Identifying Forest Loss Areas Using Google Earth Engine Coding System in Keonjhar, Odisha, India. In Asian Conference on Remote Sensing 2018. ACRS.

Jena, R., Pradhan, B., Beydoun, G., Sofyan, H. \& Affan, M. (2019). Integrated model for earthquake risk assessment using neural network and analytic hierarchy process: Aceh province, Indonesia. Geoscience Frontiers, https://doi.org/10.1016/j.gsf.2019.07.006.

Jensen, J.R., \& Cowen, D.C. (1999). Remote sensing of urban/suburban infrastructure and socioeconomic attributes. Photogrammetric Engineering and Remote Sensing, 65, 611-622.

Kalliola, R., \& Syrjanen, K. (1991). To what extent are vegetation types visible in satellite imagery?. In Annales Botanici Fennici (pp. 45-57). The Finnish Botanical Publishing Board.

Kettig, R.L., \& Landgrebe, D.A. (1976). Classification of multispectral image data by extraction and classification of homogeneous objects. IEEE Transactions on Geoscience Electronics, 14(1), 19-26. 
Lee, H.Y., Kim, T., Park, W., Lee, H.K. (2003). Extraction of digital elevation models from satellite stereo images through stereo matching based on epipolarity and scene geometry. Image and Vision Computing, 21(9), 789-796.

Lefsky, M. A., Cohen, W. B., Parker, G. G., \& Harding, D. J. (2002). Lidar remote sensing for ecosystem studies: Lidar, an emerging remote sensing technology that directly measures the three-dimensional distribution of plant canopies, can accurately estimate vegetation structural attributes and should be of particular interest to forest, landscape, and global ecologists. AIBS Bulletin, 52(1), 19-30.

Liu, L., Coops, N. C., Aven, N. W., \& Pang, Y. (2017). Mapping urban tree species using integrated airborne hyperspectral and LiDAR remote sensing data. Remote Sensing of Environment, 200, 170-182.

Low, N. (2005). The green city: Sustainable homes, sustainable suburbs. UNSW Press.

Lu, G.Y. \& Wong, D.W. (2008). An adaptive inverse-distance weighting spatial interpolation technique. Computers \& Geosciences, 34(9), 1044-1055.

Miller, R. W., Hauer, R. J., \& Werner, L. P. (2015). Urban forestry: planning and managing urban greenspaces. Waveland Press.

McPherson, E. G., Simpson, J. R., Xiao, Q., \& Wu, C. (2011). Million trees Los Angeles canopy cover and benefit assessment. Landscape and Urban Planning, 99(1), 40-50.

Mahmoud, A., Elbialy, S., Pradhan, B., \& Buchroithner, M. (2011). Field-based landcover classification using TerraSAR-X texture analysis. Advances in Space Research, 48(5), 799 805.

Mojaddadi, H., Pradhan, B., Nampak, H., Ahmad, N., \& Ghazali, A.H. (2017). Ensemble machinelearning-based geospatial approach for flood risk assessment using multi-sensor remotesensing data and GIS. Geomatics, Nat. Hazards and Risk, 8(2), 1080-1102, DOI: 10.1080/19475705.2017.1294113Naghibi, S.A., Moghaddam, D.D., Kalantar, B., Pradhan, B., \& Kisi, O. (2017). A comparative assessment of GIS-based data mining models and a novel ensemble model in groundwater well potential mapping. Journal of Hydrology, 548, 471-483. https://doi.org/10.1016/j.jhydrol.2017.03.020

Nasirzadeh, M., Khazael, A. A., \& bin Khalid, M. (2010, July). Woods recognition system based on local binary pattern. In Computational Intelligence, Communication Systems and Networks (CICSyN), 2010 Second International Conference on (pp. 308-313). IEEE. 
Ojala, T., Pietikainen, M., \& Maenpaa, T. (2002). Multiresolution gray-scale and rotation invariant texture classification with local binary patterns. IEEE Transactions on Pattern Analysis and Machine Intelligence, 24(7), 971-987.

Oostdijk, A., Persie, M.V., Noorbergen, H.H.S., \& Van Rijn,J.W. (2008). Multi scale object based detection and classification of roads and vehicles in high-resolution optical satellite imagery.

Pradhan, B., Moneir, A.A.A. \& Jena, R., (2018). Sand dune risk assessment in Sabha region, Libya using Landsat 8, MODIS, and Google Earth Engine images. Geomatics, Natural Hazards and Risk, 9(1), 1280-1305.

Pirotti, F. (2010). Assessing a template matching approach for tree height and position extraction from lidar-derived canopy height models of pinus pinaster stands. Forests, 1(4), 194-208.

Qi, X., Shen, L., Zhao, G., Li, Q., \& Pietikäinen, M. (2015). Globally rotation invariant multi-scale co-occurrence local binary pattern. Image and Vision Computing, 43, 16-26.

Roberts, L. (2011). 9 Billion?. Science, 333(6042), 540-543.

Ryherd, S., \& Woodcock, C. (1996). Combining spectral and texture data in the segmentation of remotely sensed images. Photogrammetric Engineering and Remote Sensing, 62(2), 181194.

Schreyer, J., Tigges, J., Lakes, T., \& Churkina, G. (2014). Using airborne LiDAR and QuickBird data for modelling urban tree carbon storage and its distribution-A case study of Berlin. Remote Sensing, 6(11), 10636-10655.

Shackelford, A.K., \& Davis, C.H. (2003). A combined fuzzy pixel-based and object-based approach for classification of high-resolution multispectral data over urban areas. IEEE Transactions on Geoscience and Remote sensing, 41(10), 2354-2363.

Sibaruddin, H.I., Shafri, H.Z.M., Pradhan, B., \& Haron, N.A. (2018). Comparison of pixel-based and object-based image classification techniques in extracting information from UAV imagery data. In IOP Conference Series: Earth and Environmental Science, 169(1), 012098. IOP Publishing.

Tehrany, M.S., Pradhan, B., \& Jebuv, M.N. (2014). A comparative assessment between object and pixel-based classification approaches for land use/land cover mapping using SPOT 5 imagery. Geocarto International, 29(4), 351-369.

Wen, D., Huang, X., Liu, H., Liao, W., \& Zhang, L. (2017). Semantic classification of urban trees using very high resolution satellite imagery. IEEE Journal of Selected Topics in Applied Earth Observations and Remote Sensing, 10(4), 1413-1424 
Yang, W.Q., Spink, D.M., York, T.A. and McCann, H. (1999). An image-reconstruction algorithm based on Landweber's iteration method for electrical-capacitance tomography. Measurement Science and Technology, 10(11), 1065.

Yu, Q., Gong, P., Clinton, N., Biging, G., Kelly, M., \& Schirokauer, D. (2006). Object-based detailed vegetation classification with airborne high spatial resolution remote sensing imagery. Photogrammetric Engineering \& Remote Sensing, 72(7), 799-811.

Zhang, C., Zhou, Y., \& Qiu, F. (2015). Individual tree segmentation from LiDAR point clouds for urban forest inventory. Remote Sensing, 7(6), 7892-7913. 